\title{
Stage IA Bone Cancer AJCC v8
}

National Cancer Institute

\section{Source}

National Cancer Institute. Stage IA Bone Cancer A/CC v8. NCI Thesaurus. Code C136614.

Stage IA includes: T1, N0, M0, G1 or GX. T1: Tumor measuring $8 \mathrm{~cm}$ or less in greatest

dimension. N0: No regional lymph node metastasis. M0: No distant metastasis. GX:

Grade cannot be assessed. G1: Well differentiated, low grade. (AJCC 8th ed.) 